\title{
Xylitol Chewing Gums and Caries Rates: A 40-month Cohort Study
}

\author{
K.K. Mäkinen*, C.A. Bennett ${ }^{1}$, P.P. Hujoel ${ }^{2}$, P.J. Isokangas ${ }^{3}$, K.P. Isotupa ${ }^{4}$, H.R. Pape, Jr., and P.L. Mäkinen \\ Department of Biologic and Materials Sciences, School of Dentistry, University of Michigan, Ann Arbor, Michigan 48109-1078; ${ }^{1}$ Belize City \\ Hospital, Belize City, Belize; ${ }^{2}$ Department of Dental Public Health Sciences, University of Washington, Seattle; ${ }^{3}$ Ylivieska Health Center, \\ Ylivieska, Finland; and ${ }^{4}$ Institute of Dentistry, University of Turku, Turku, Finland; *to whom correspondence should be addressed
}

\begin{abstract}
Dental caries is a pandemic infectious disease which can affect the quality of life and consumes considerable health care resources. The chewing of xylitol, sorbitol, and even sugar gum has been suggested to reduce caries rates. No clinical study has simultaneously investigated the effectiveness of these gums when compared with a group receiving no chewing gum. A 40-month double-blind cohort study on the relationship between the use of chewing gum and dental caries was performed in 1989-1993 in Belize, Central America. One thousand two hundred and seventy-seven subjects (mean age, 10.2 years) were assigned to nine treatment groups: one control group (no supervised gum use), four xylitol groups (range of supervised xylitol consumption: 4.3 to $9.0 \mathrm{~g} /$ day), two xylitolsorbitol groups (range of supervised consumption of total polyols: 8.0 to $9.7 \mathrm{~g} /$ day), one sorbitol group (supervised consumption: $9.0 \mathrm{~g} /$ day), and one sucrose group ( $9.0 \mathrm{~g} /$ day). The gum use during school hours was supervised. Four calibrated dentists performed the caries registrations by means of a modified WHO procedure. The primary endpoint was the development of an unequivocal caries lesion on a non-cavitated tooth surface. Compared with the no-gum group, sucrose gum usage resulted in a marginal increase in the caries rate (relative risk, 1.20; $95 \%$ confidence interval, 0.96 to $1.49 ; \mathrm{p}=0.1128$ ). Sorbitol gum significantly reduced caries rates (relative risk, $0.74 ; 95 \%$ confidence interval, 0.6 to $0.92 ; p=0.0074$ ). The four xylitol gums were most effective in reducing caries rates, the most effective agent being a 100\% xylitol pellet gum (relative risk, $0.27 ; 95 \%$ confidence interval, 0.20 to $0.36 ; p=0.0001$ ). This gum was superior to any other gum $(p<0.01)$. The xylitolsorbitol mixtures were less effective than xylitol, but they still reduced caries rates significantly compared with the no-gum group. DMFS analyses were consistent with these conclusions. The results suggest that systematic usage of polyol-based chewing gums reduces caries rates in young subjects, with xylitol gums being more effective than sorbitol gums.
\end{abstract}

Key words: Xylitol, sorbitol, sugar, chewing gum, caries increments.

Received February 8, 1995; Accepted August 31, 1995

\section{Introduction}

Despite widespread exposure to diverse forms of fluoride, dental caries remains a staggering public health (Miller et al., 1987; Brunelle, 1989) and economic burden in the USA. More than $\$ 37$ billion was spent on dental services in the USA in 1992 (American Dental Association, 1992; Burner et al., 1992), far more than half of which was for the prevention of tooth decay, single-tooth restoration, and extraction and replacement of teeth. Increasing evidence indicates the emergence of dental caries as a health problem in the developing world (World Health Organization, 1989; Stephen, 1993).

During the past 20 years, several reports have indicated that high-content xylitol confections, including candies and chewing gums, are non-cariogenic and may actually inhibit caries. Studies have examined DMFS scores as a function of the effect of sorbitol gum compared with no gum (Möller and Poulsen, 1973; Glass, 1981); xylitol gum compared with no gum and with topical fluoride (Scheinin et al., 1985a,b; Kandelman et al., 1988; Isokangas et al., 1988; Kandelman and Gagnon, 1990), and xylitol gum compared with sucrose (Scheinin et al, 1975). Xylitol has been shown consistently to reduce DMFS scores, while the effect of sorbitol has been equivocal (Möller and Poulsen, 1973; Glass, 1981; Birkhed, 1994). However, owing to the use of diverse control groups (no gum, sugar gum, fluoride treatment program), no simultaneously collected data from a single study are available on the association of xylitol gum with caries, by comparison with sorbitol and sucrose gum. Furthermore, the higher manufacturing costs of xylitol when compared with sorbitol necessitated a study where various combinations of these polyols could be investigated; partial substitution of sorbitol with xylitol may keep manufacturer's costs down while still providing optimal dental benefit. Consequently, a simultaneous comparison of the cariologic significance of chewing gums containing either xylitol, sorbitol, two different mixtures of xylitol and 
sorbitol, or sucrose, by comparison with no supplied gum, was undertaken and is reported herein. This study was carried out in Belize, Central America.

Belize is a democratically governed nation of about 200,000 inhabitants. Belize City is an urban district of about 45,000 inhabitants (in 1991). The children are $68 \%$ Creole, $10 \%$ Mestizo, $6 \%$ Garifuna, and 7\% other races (Belize Ministry of Finance-Central Statistical Office, 1991). Belize's economy is agricultural, with the cultivation and export of cane sugar and citrus products playing an important part. Carbohydrates and starchy foods make up the bulk of the diet (Belize Ministry of Finance-Central Statistical Office, 1991); the average per capita consumption of domestically produced sucrose was 40 to $55 \mathrm{~kg}$ in 1989-1993 (when this study was carried out), about $33 \%$ to $75 \%$ higher than the amounts consumed in the USA (Glinsmann et al., 1986; Licht, 1993; Woodward and Walker, 1994). The average frequency of intake of sweet items, aside from chewing gum, was surveyed on three occasions in this study and was found to range between 12 and 14 per day. No attempt was made to affect the subjects' dietary regimen or oral hygiene practices during this study, other than to supply/not supply chewing gums for use according to specific protocol. Caries prevalence in Belize City is reported to be high (World Health Organization, 1984, 1989; Bennett, 1989; Woodward and Walker, 1994). Most children in Belize City use toothbrushes and fluoride toothpaste, but the technique and time spent are uncertain and inconsistent. Drinking water in Belize is not fluoridated. The above information suggests that the oral health of young subjects in Belize is influenced by several caries-predisposing factors. The goal of the present study was to investigate the effect of chewing-gum usage on the caries rate in initially 10-year-old subjects living in the Belize City district of Belize.

\section{Materials and methods}

\section{Subjects and study design}

This study was approved by the Human Subjects Committee of the University of Michigan Dental School and by the Ministry of Health of Belize. Participation in the program was voluntary. All families solicited for consent for the participation of their children, except one, consented, and all of these children assented. No child was asked to leave the program during its implementation.

A double-blind cohort study was conducted to evaluate the effects of six chewing gums and three modes of usage on cariesonset rates. The primary null hypothesis was that the choice and amount of bulk sweetener included in six different chewing gums consumed by a population with high caries prevalence would have no effect on the risk for a caries onset on a sound tooth surface, in comparison with "no gum" use. The sample size and study duration were based on the investigators' experience in conducting analogous trials, and on practical limitations within Belize City. All 4th grade pupils $(n=1277$; average age, 10.2 years) attending any of the 19 public schools of Belize City, Belize, in September, 1989, were recruited to this study. There were no other inclusion or exclusion criteria.

The study gums had consumer-approved and palatable composition, and were either identical or similar to brands marketed in Europe and North America. They were manufactured to have, as closely as possible, comparable aggregate carbohydrate/sugar alcohol (polyol) content, physical and taste properties, and were formulated according to current manufacturing art; some gums were current items of commerce, as stipulated below. In some cases, the chewing gums were used at two different frequencies/day ( 3 or 5 times) or in two different physical forms, stick ( $s$ ) or pellet (p). One stick-shaped gum or two pellet-shaped gums were chewed at

Table 1. Designation and description of experimental groups and chewing gums, and use patterns of gums by subjects

\begin{tabular}{|c|c|c|c|c|c|c|c|c|c|c|}
\hline \multirow[b]{2}{*}{$\begin{array}{l}\text { Group } \\
\text { Number }\end{array}$} & \multirow[b]{2}{*}{$\begin{array}{l}\text { Abbreviation } \\
\text { Used in Text }\end{array}$} & \multirow{2}{*}{\multicolumn{2}{|c|}{$\begin{array}{l}\text { Number of Pieces } \\
\text { Used/Day (No. of } \\
\text { chewing episodes } \\
\text { per day) }\end{array}$}} & \multicolumn{7}{|c|}{ Amount of Sweetener Present in Gum Pieces/Day $(\mathrm{g})$} \\
\hline & & & & Suc & $\begin{array}{l}\text { Corn } \\
\text { Swtnr. }\end{array}$ & Xyl & Sorb & Lyc $^{\mathrm{e}}$ & $\begin{array}{l}\text { Total } \\
\text { Polyol }\end{array}$ & $\begin{array}{l}\text { Total } \\
\text { Sugars }\end{array}$ \\
\hline 1 & No gum ${ }^{c}$ & - & $(-)$ & - & - & 一 & - & 一 & - & 一 \\
\hline 2 & Sugar-s5 & 5 & (5) & 9.0 & $1.3^{\mathrm{d}}$ & - & - & 0 & 0 & 10.3 \\
\hline 3 & Sorb-p5 & 10 & (5) & 0 & 0 & - & 9.0 & 0 & 9.0 & 0 \\
\hline 4 & $3: 2 X S-p 5$ & 10 & (5) & 0 & 0 & 5.9 & 3.8 & 0 & 9.7 & 0 \\
\hline 5 & $1: 3$ XS-p5 & 10 & (5) & 0 & 0 & 2.0 & 6.0 & 0 & 8.0 & 0 \\
\hline 6 & Xyl-s3 & 3 & (3) & 0 & 0 & 5.4 & 0 & 0.7 & 6.1 & 0 \\
\hline 7 & Xyl-s5 & 5 & (5) & 0 & 0 & 9.0 & 0 & 1.3 & 10.3 & 0 \\
\hline 8 & Xyl-p3 & 5 & (3) & 0 & 0 & 4.3 & 0 & 0 & 4.3 & 0 \\
\hline 9 & Xyl-p5 & 10 & (5) & 0 & 0 & 8.5 & 0 & 0 & 8.5 & 0 \\
\hline
\end{tabular}

a Suc, sucrose; Xyl or X, xylitol; Sorb or S, sorbitol; Lyc, Lycasin ${ }^{\circledR}$. Physical form of gum, i.e., stick (s) or pellet (p), and number of chewing episodes per day are indicated after hypen; thus, $-\mathrm{s} 5$ means five episodes of chewing a stick gum.

$b \quad$ In pellet gum groups, two pellets $(2 \times 1.3 \mathrm{~g})$ were used at a time, except that one pellet was chewed at the third episode of the Xyl-p3 group. In stick gum groups, one stick $(2.8 \mathrm{~g})$ was used at a time.

c Denotes "no gum as part of the program". It is unknown how much sugar, in gum or other forms, was used by children in the "No gum" group, or by children in Groups 2 to 9 , aside from the quantities in gums provided by the study. It is known, however, that there is essentially no (commercially available) xylitol, sorbitol, or Lycasin ${ }^{\circledR}$ in the Belizian diet. Hence, the values of " 0 " gums can be stipulated with relative certainty.

d Corn sweetener composed of $55 \%$ fructose and $45 \%$ glucose was included, as solids, in the indicated amount.

e Lyc, Lycasin ${ }^{\circledast}$, is composed of $8 \%$ sorbitol, $52.5 \%$ maltitol, and $39.2 \%$ hydrogenated starches. It was included, as solids, in the indicated amount, which reflects the fermentable sorbitol and maltitol portions only. 
Table 2. Demographic and clinical information about the groups at baseline for 1277 subjects

\begin{tabular}{|c|c|c|c|c|c|c|c|}
\hline $\begin{array}{l}\text { Group } \\
\text { No. }\end{array}$ & $\begin{array}{l}\text { Abbreviation } \\
\text { Used in Text }\end{array}$ & $\begin{array}{l}\text { No. of } \\
\text { Schools }^{b}\end{array}$ & $\begin{array}{l}\text { Gender } \\
\text { (\% males) }\end{array}$ & $\begin{array}{c}\text { Average } \\
\text { Age } \\
\text { (years) }\end{array}$ & $\begin{array}{l}\text { Avg. No. of Sound } \\
\text { Surfaces/Subject }\end{array}$ & $\begin{array}{l}\text { Avg. No. of } \\
\text { Decayed, Missing } \\
\text { \& Filled Surfaces } \\
\text { per Subject }\end{array}$ & $\begin{array}{l}\text { Evidence } \\
\text { for Dental } \\
\text { Treatment }\end{array}$ \\
\hline 1 & No gum (143) & 1 & 45.5 & $10.2(1.4)^{\mathrm{d}}$ & $84.0(25.3)$ & $4.8(5.4)$ & $8(5.6)$ \\
\hline 2 & Sugar-s5 (137) & 1 & 50.7 & $10.1(1.1)$ & $74.3(24.6)$ & $4.8(5.3)$ & $19(13.9)$ \\
\hline 3 & Sorb-p5 (136) & 1 & 56.6 & $10.1(0.6)$ & $76.3(24.1)$ & $5.4(4.9)$ & $22(16.2)$ \\
\hline 4 & $3: 2$ XS-p5 (140) & 3 & 53.2 & $10.1(1.1)$ & $77.8(25.8)$ & $5.1(4.8)$ & $17(12.1)$ \\
\hline 5 & 1:3 XS-p5 (141) & 2 & 59.0 & $9.8(1.0)$ & $73.8(26.3)$ & $4.9(5.0)$ & $10(7.1)$ \\
\hline 6 & Xyl-s3 (157) & 3 & 56.1 & $10.9(0.9)$ & $93.6(24.0)$ & $6.6(6.2)$ & $20(12.7)$ \\
\hline 7 & Xyl-s5 (137) & 2 & 47.4 & $10.5(0.8)$ & $84.7(24.2)$ & $6.8(5.8)$ & $27(19.7)$ \\
\hline 8 & Xyl-p3c (148) & 4 & 64.2 & $9.9(1.2)$ & $73.1(26.6)$ & $4.0(5.1)$ & $10(6.8)$ \\
\hline 9 & Xyl-p5 $5^{c}(138)$ & 2 & 51.4 & $10.0(1.2)$ & $75.5(25.2)$ & $5.7(5.2)$ & $23(16.7)$ \\
\hline
\end{tabular}

a Physical form of gum, i.e., stick (s) or pellet (p), and number of chewing episodes per day are indicated after hyphen; thus, -s5 means five episodes of chewing a stick gum. The figures in parentheses show the number of subjects at baseline.

b Number of schools that provided the study subjects in each group.

c Marketed by Leaf Group B.V. as XyliFresh 100 and XyliFresh Professional.

d Values are expressed as means, and standard deviations are indicated in parentheses.

e Number (percentage) of subjects who had fillings and extractions.

each episode, except that when gum was used three times per day, only one pellet was chewed at the last episode (this concerns only group no. 8 below; Table 1). Nine chewing gum groups, numbered as follows and referred to in text and tables by the parenthetical abbreviations, were studied (Table 1): (1) "No gum"; (2) $\sim 60 \%$ sucrose/ $8 \%$ corn sweetener stick gum 5 times per day (Sugar-s5; "sucrose gum"); (3) $\sim 65 \%$ sorbitol pellet gum 5 times per day (Sorb-p5; "sorbitol gum"); (4) $\sim 45 \%$ xylitol/30\% sorbitol pellet gum 5 times per day (3:2 XS-p5); (5) $\sim 15 \%$ xylitol $/ 45 \%$ sorbitol pellet gum 5 times per day (1:3 XSp5); (6) $\sim 60 \%$ xylitol $/ 9 \%$ Lycasin ${ }^{\circledR}$ stick gum 3 times per day (Xyl-s3); (7) $\sim 60 \%$ xylitol/9\% Lycasin ${ }^{\circledR}$ stick gum 5 times per day (Xyl-s5); (8) $~ 65 \%$ xylitol pellet gum 3 times per day (Xyl-p3); and (9) $\sim 65 \%$ xylitol pellet gum 5 times per day (Xyl-p5). The amounts of sugars [sucrose, glucose, and fructose (the latter two provided $45 \%$ glucose $/ 55 \%$ fructose by the corn syrup; Corn Products, Inc., Summit-Argo, IL)] and of polyols (xylitol and sorbitol; Xyrofin, Schaumberg, IL) and Lycasin(B) (Roquette Frêres, France; a hydrogenated partial hydrolyzate of starch containing about $8 \%$ free sorbitol and $52.5 \%$ free maltitol) which are contained in the study chewing gums, and the amounts provided by each gum product per day of supervised use, are detailed in Table 1. Further formulation details are available upon request.

Because of the problem of subject exchange of test items in school-based studies, chewing gums were not randomly provided to children within schools. Rather, a specific chewing gum was assigned to each school, with the result that the entire 4th grade class within any one school was given the same gum and protocol, consistent with community-based health intervention programs. Before baseline examinations in September, 1989, the 1277 fourth-graders were divided into nine convenience groups of 136 to 157 subjects, so that each school's entire eligible student population was assigned to one of the groups. In some groups, the study subjects were composed of the entire 4th grade class of several smaller schools (Table 2).

\section{Blinding and study implementation}

The gums (pellet- and stick-shaped) were packed in number-coded blank wraps ( 5 pieces/package). These were, in turn, packed in number-coded boxes on which the schools' names were marked. The quality and the composition of the gums were checked with samples from the gum lots, both at the manufacturing plant and at the gum storage area in Belize City. Throughout the study, participating pupils, their parents or guardians, and the teachers, principals and other school and health-care authorities, and the examining clinicians were not aware of the type of gum used by any individual or by any school, nor were they informed about the number of experimental groups included. Periodic checking of the subjects', teachers', and families' awareness of the details of the program confirmed that while its significance was comprehended, the effectiveness of the masking was not compromised. The subjects were bussed or transported with private cars to the clinic from the 19 participating schools (and occasionally from home residences). The busses were hired from a commercial bus line; the busses had no sign indicating from which school the subjects arrived. All transportation was accomplished in a random order which varied at all examinations. The blinded caries raters were not aware of the order in which the examination cohorts arrived at the examination clinic; subjects from several groups could be simultaneously present. The local author (CAB) visited schools regularly to check gum deliveries and gum usage, while another author (KKM) made 32 monitoring site visits to verify the delivery of gums to schools and subjects, confirm their correct use, meet with school personnel and subjects, and ensure the general implementation of the program. An independent clinical study monitor (Brogle Associates, Upper Montclair, NJ) also assessed the integrity of the program (10 site visits). 


\section{Gum use and instructions}

On 200 school days/year, five-minute periods of gum use were supervised by class teachers who were supplied with mechanical timers. On non-school days, partial supervision was provided by parents or guardians. Those who chewed gum three times daily in school did so at 8:30, 11:30, and 14:30 hours, while those who chewed gum five times daily did so at 8:30, 10:00, 11:30, 13:00, and 14:30 hours. The 13:00 and 14:30 times are after the mid-day meal. On Fridays, and on the day before holidays and vacations, the children received the appropriate measured amounts of gum from the class teacher, to enable them to maintain daily gum usage near the level supervised on schooldays. Supplies for weekends and holidays/vacations were $50 \%$ larger/day than on school days, because it was assumed that family members might explore use of the gums. Additionally, before Easter and Christmas holidays (two and three weeks, respectively), and before summer vacations (two months), teachers instructed the children verbally to continue the same chewing practice at home as used in school, this being reinforced by written instructions from schools sent during the holiday/vacation periods. No written or other instructions were sent to families whose children belonged to the no-gum group. This gum usage program was not presented as a replacement of other gum or candy use. No commercial xylitol product was available in Belize, and the consumption of sorbitol-containing commercial products was uncommon.

\section{Assessment of response variable}

The primary pre-trial specified response variable was the onset of a caries lesion on a previously sound or unerupted surface. No information on caries experience and age was available at the time the groups were formed. The clinical assessments were carried out by four experienced, calibrated, and blinded dentists at baseline, 16, 28, and 40 months. At baseline, subjects were systematically and blindly assigned to examiners. This subject-examiner assignment was fixed for the duration of the program. A clinic with four identical examination rooms and equipment (dental chairs, lighting, compressed air) was built for the program's purposes, and 200 explorers were purchased and inspected microscopically at each examination period for sharpness and integrity (Pape and Mäkinen, 1994).

Each examiner scored the teeth according to the definitions of WHO codes (World Health Organization, 1979), with the exception that WHO code 0 (sound surface) and WHO code 1 (white spot lesions and enamel and dentin surfaces unpenetrable to a sharp explorer) were regarded as sound surfaces and coded Do. Enamel caries with clinically detectable loss of substance, usually represented by early pit and fissure caries with softness at the base of the pit near the dentino-enamel junction (WHO code 2; enamel caries with clinically detectable loss of substance), was labeled as Ds, i.e., essentially equivalent to a Radike sticky fissure (Radike, 1972; Charbenau, 1988). Caries lesions associated with fracture of the surrounding enamel owing to undermining by carious dentin (WHO code 3 ; dentin caries) were labeled Dm. Severe lesions with probable pulpal involvement and shelling out of virtually the entire structure of the tooth (WHO code 4;

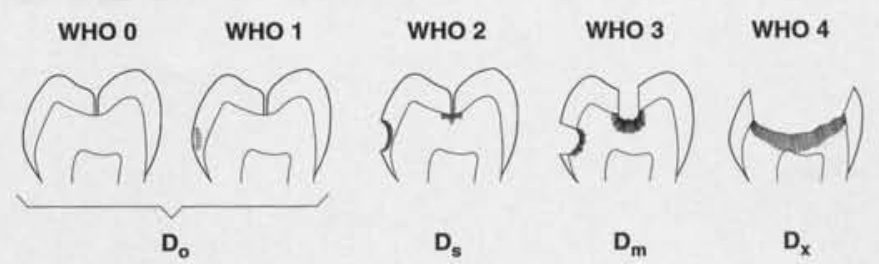

Figure 1. Description of caries coding used in the study as compared with WHO coding.

deep cavity with probable pulp involvement) were labeled Dx. A diagrammatic representation of such lesion categories is presented by the Fig. Molars and premolars were divided into five surfaces, and incisors and canines into four. Surfaces with sealants (which were rare) were scored sound (Do). Four surfaces for anterior or five surfaces for posterior teeth were scored in a specified category for traumatic or orthodontic loss (the latter was extremely rare). Surfaces of teeth missing apparently because of caries were coded as extracted according to WHO. If a Do surface developed cavitation or was filled or extracted, it was taken as evidence of caries lesion onset between examinations. The primary outcome variable was the development of an unequivocal cavity on a previously structurally intact surface. As the study progressed, it was apparent that some lesions which were grossly cavitated, and thus coded Dm or Dx, became hard. Clinicians coded them as Do, which was re-coded as Dh ("remineralized" or "arrested"). For the purpose of $\triangle D M F S$ calculations (vide infra), these surfaces were regarded as decayed. Ds lesions that became hard were coded Do. The changes from Ds to Do were included in the DMFS analyses (vide infra), and sometimes caused the $\triangle \mathrm{DMFS}$ to become negative.

The threshold for scoring a lesion was an established physical discontinuity and softness of the enamel in either fissures or pits or smooth surfaces in the gingival third of teeth (less common). Such lesions approach the dentin (Charbeneau, 1988; Newbrun, 1989; Sturdevant, 1995). For an enamel caries lesion to be called Ds, there had to be an actual surface break of the enamel (Fig.), and the base of the lesion had to be soft instead of giving only a "catch" to the sharp caries explorer (Radike, 1972: Charbeneau, 1988). Areas which showed some loss of surface or gave a "catch" to the explorer, but which did not include any softness at the base of the lesion, were scored as Do at all times of the study, i.e., at the baseline and at subsequent examinations. No dental radiographs were taken or used in this study.

\section{Calibration of examiners; examiner error analyses}

The four caries raters were calibrated prior to the four examinations carried out at baseline and at 16,28 , and 40 months. Each time, the calibrations included a discussion and a review of the caries concepts (Fig.), and a "practice" on 8 to 10 randomly chosen study subjects one day before the commencement of the examination sessions proper. In these practice sessions, the four examiners worked as one group. The analyses of the inter-examiner error proper were then carried out according to Landis and Koch (1977) on the following number of randomly chosen subjects: baseline $=32,16$ months $=30,28$ 
Table 3. Descriptive statistics on the number of caries lesion onsets observed among those 1135 subjects who were seen on at least two examination dates

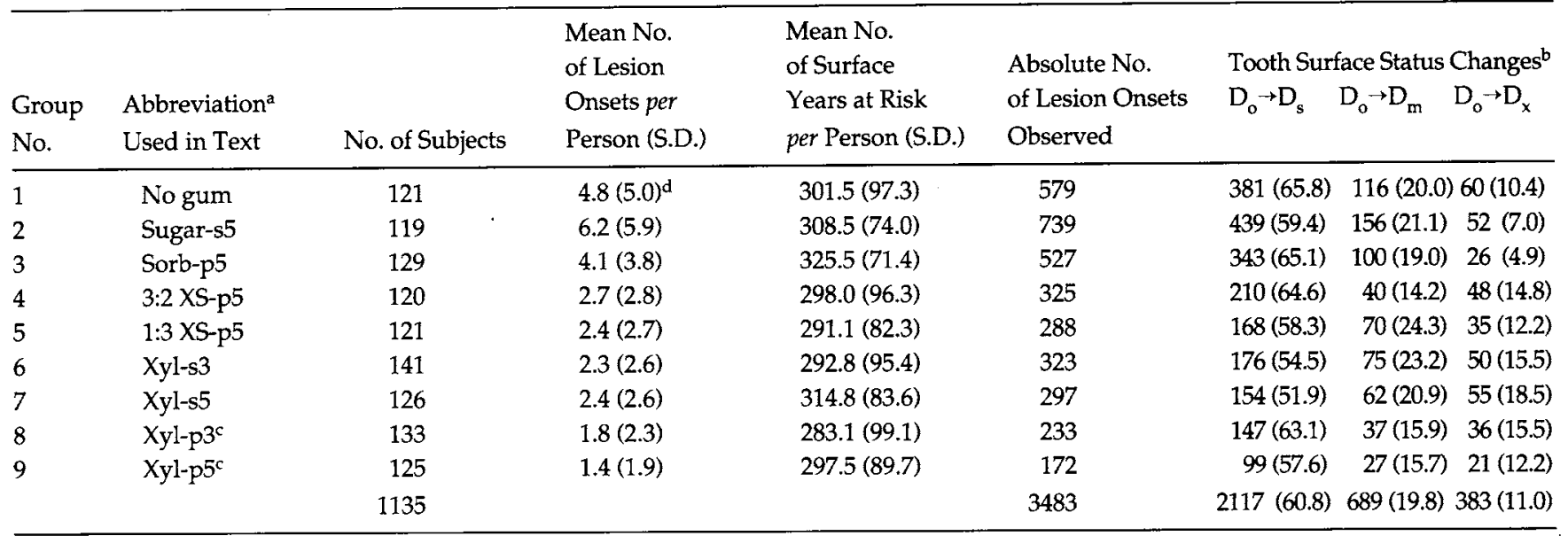

a Physical form of gum, i.e., stick (s) or pellet (p), and number of chewing episodes per day are indicated after hyphen; thus, -s5 means five episodes of chewing a stick gum.

b The absolute number of tooth surfaces which were $D_{0}$ at baseline and were filled at later examination was 165; the absolute number of tooth surfaces which were $D_{0}$ at baseline and which were extracted at later examination was 129 . These accounted for $8.4 \%$ of the $D_{0}$ to $>D_{0}$ changes and, for simplicity, are not shown in the Table.

c Marketed by Leaf Group B.V. as XyliFresh 100 and XyliFresh Professional.

d Values are expressed as means, and standard deviations are indicated in parentheses.

months $=28$, and 40 months $=26$. The intra-examiner error analyses were carried out according to Shaw and Murray (1975) on the above study subjects at two- or three-week intervals at each of the four examinations. In this analysis, the values of $r$ and $P$ were calculated; $r=a / b$ and $P=b /(a+b)$, where $a=$ number of sites with disagreements as to sound or carious, and $b$ $=$ number of sites consistently diagnosed as carious.

\section{Data management and statistical analysis}

The primary purpose of the statistical analysis was to assess whether the rates of caries onset differed among groups. Rates were estimated by standard epidemiological methods which incorporate the time at risk (Breslow and Day, 1980; Hujoel et al., 1994). Each surface diagnosed as structurally intact at baseline (or which erupted during the study) and which was examined on at least one other examination date was considered at risk for lesion development. In the analysis, the rate at which non-cavitated surfaces $\left(D_{0}\right)$ converted to caries lesions (with clinically detectable loss of substance; codes $D_{s^{\prime}}$ $\mathrm{D}_{\mathrm{m}^{\prime}}$ or $\mathrm{D}_{\mathrm{x}^{\prime}}$ Fig.), fillings, or extractions was estimated. Subjects stopped contributing surface-time at risk when they were last examined. If a surface did not develop a cavity between two examinations, the amount of observation time contributed by that surface equaled the time between the two examinations. If the surface developed a lesion during a time interval, it was assumed that the caries onset occurred in the middle of the interval (Hujoel et al., 1994). Thirty-one surfaces coded as having a filling unrelated to caries (WHO code 9) were deleted from the analysis. Their inclusion or exclusion had no effect on the reported statistics.

The caries onset rates were estimated by means of Poisson regression models within the framework of generalized estimating equations to account for the correlated observations within the same subjects (Liang and Zeger, 1986; Karim and Zeger, 1988). The goal was to provide the best defense of the lack of association between the chewing gum regimens and caries onsets (Maclure, 1990). The analyses were adjusted for potentially confounding variables determined at baseline, including gender, age, caries experience at baseline (DMFS score), and the number of sound surfaces. The analyses could not be adjusted for the school factor because certain gums were chewed at one school only. As a result, the school and treatment factors were intrinsically aliased (McCullagh and Nelder, 1989). The statistical significance of regression parameters was assessed by the Wald statistic, the division of the maximum likelihood estimate of the slope parameter by an estimate of its standard error. Both significance tests and confidence intervals were based on a robust estimate of the standard error of the regression coefficient. Because the comparisons between chewing gum groups were pre-planned, no adjustments for multiple comparisons were performed.

So that the effect of dropout bias would be minimized, all subjects seen on at least two of the visits were included in the analysis (Louis, 1988). The reasons for dropping out of the study, according to teachers, were as follows: moved and could not be located $(39.7 \%)$, emigrated $(20.9 \%)$, repeated the class and discontinued gum use (9.5\%), dropped out of school (4.0\%), absent from the school at the 40 -month examination $(6.9 \%)$, long-term hospitalization (two subjects), jailed (one subject), and deceased (one subject). The remainder (19.0\%) of the dropouts discontinued the program for unknown reasons.

The difference between 40-month and baseline DMFS scores is also presented. The results of these analyses must be interpreted differently from the Poisson regression models 
Table 4. Changes from baseline to endpoint ( 40 months) in caries rate scores and relative risk (RR) estimates ${ }^{\mathrm{a}}$

\begin{tabular}{|c|c|c|c|c|c|c|c|}
\hline $\begin{array}{l}\text { Group } \\
\text { Number }\end{array}$ & $\begin{array}{l}\text { Abbreviation } \\
\text { Used in Text }\end{array}$ & $\begin{array}{l}\text { Crude } \\
\text { Caries } \\
\text { Rates }^{c}\end{array}$ & Crude RR & $\begin{array}{l}\text { RR Adjusted } \\
\text { for Gender }\end{array}$ & $\begin{array}{l}\text { RR Adjusted } \\
\text { for Age and } \\
\text { Gender }\end{array}$ & $\begin{array}{l}\text { RR Adjusted for } \\
\text { Age, Gender } \\
\text { and DMFSd }\end{array}$ & $\begin{array}{l}\text { RR Adjusted } \\
\text { for Age, Gender, } \\
\text { DMFS, and } \\
\text { Number of Sound } \\
\text { Surfaces at Baseline }\end{array}$ \\
\hline 1 & No gum & $15.9(13.2-19.1)$ & & & & & \\
\hline 2 & Sugar-s5 & $20.1(17.0-23.9)$ & $1.27(0.99-1.63)$ & $1.28(1.00-1.63)$ & $1.26(0.99-1.61)$ & $1.22(0.98-1.53)$ & $1.20(0.96-1.49)$ \\
\hline 3 & Sorb-p5 & $12.6(10.7-14.7)$ & $0.79(0.62-1.01)$ & $0.80(0.63-1.02)$ & $0.79(0.63-1.00)$ & $0.75(0.61-0.93)$ & $0.74(0.60-0.92)$ \\
\hline 4 & 3:2 XS-p5 & $9.1(7.6-10.9)$ & $0.57(0.44-0.74)$ & $0.58(0.45-0.75)$ & $0.58(0.45-0.74)$ & $0.57(0.45-0.72)$ & $0.56(0.44-0.71)$ \\
\hline 5 & $1: 3$ XS-p5 & $8.2(6.7-10.0)$ & $0.50(0.39-0.68)$ & $0.53(0.40-0.69)$ & $0.51(0.39-0.67)$ & $0.50(0.38-0.67)$ & $0.49(0.38-0.65)$ \\
\hline 6 & Xyl-s3 & $7.8(6.4-9.5)$ & $0.49(0.38-0.64)$ & $0.50(0.38-0.65)$ & $0.52(0.40-0.67)$ & $0.48(0.37-0.61)$ & $0.48(0.37-0.61)$ \\
\hline 7 & Xyl-s5 & $7.5(6.2-9.0)$ & $0.47(0.36-0.61)$ & $0.47(0.37-0.61)$ & $0.48(0.37-0.62)$ & $0.44(0.34-0.57)$ & $0.44(0.34-0.56)$ \\
\hline 8 & Xyl-p3 ${ }^{e}$ & $6.2(4.9-7.8)$ & $0.39(0.29-0.52)$ & $0.40(0.30-0.53)$ & $0.39(0.29-0.52)$ & $0.42(0.32-0.55)$ & $0.41(0.31-0.54)$ \\
\hline 9 & $X y l-p 5^{e}$ & $4.6(3.6-6.0)$ & $0.29(0.21-0.40)$ & $0.29(0.21-0.40)$ & $0.29(0.21-0.39)$ & $0.27(0.20-0.37)$ & $0.27(0.20-0.36)$ \\
\hline
\end{tabular}

a Means and (RR) are presented as crude values, and also as values adjusted for gender; for age and gender; for age, gender, and the DMFS score; and for age, gender, DMFS score, and the number of sound surfaces at baseline. Relative risk values higher than 1 indicate an increased caries risk, a harmful effect, while relative risk values smaller than 1 indicate a decreased caries risk, a beneficial effect. The percent confidence interval expresses the $95 \%$ probability that the reported intervals include the true relative risk. This relative risk (percent confidence interval) statistic is used as a measure of treatment efficacy (Breslow and Day, 1980; Lilienfeld and Stolley, 1994).

b Physical form of gum, i.e., stick (s) or pellet (p), and number of chewing episodes per day are indicated after hyphen; thus, $-\mathrm{s} 5$ means five episodes of chewing a stick gum.

Caries lesion onsets per 1000 surfaces per year ( $95 \%$ confidence intervals).

Number of Decayed, Missing, and Filled Surfaces.

Marketed by Leaf Group B.V. as XyliFresh 100 and XyliFresh Professional. $\mathrm{n}=1135$.

(Hujoel et al., 1994). First, the DMFS analysis reflects not only the number of lesion onsets, but also the number of caries "reversals". As a result, shifts of status from Ds to Do I"healing effects"] are confounded with preventive effects (prevention of caries onsets) in the DMFS analyses. Second, because differences are taken between DMFS scores at baseline and at 40 months, only 861 subjects ( $24 \%$ less than for the calculation of incidence rates) could be included in these analyses. As a result, 783 caries onsets $(22.5 \%$ of the total number of caries onsets) and $72.2 \times 103$ surface-years $(21.1 \%$ of the total number of surface years) observed in the cohort could not be included in this DMFS analysis. The changes in DMFS score were analyzed with analysis of variance models, with $\triangle D M F S$ as the response variable and treatment groups, gender, age, and the number of sound surfaces at baseline as the explanatory variables. Hypotheses regarding the parallel slopes were examined and found to be non-significant.

Baseline differences were evaluated by the Bonferroni $t$ test, controlling for type I "experimentwise" error rate.

\section{Results}

\section{Comparisons at baseline}

The following significant differences were observed at baseline (Bonferroni $t$ test, $\mathrm{p}<0.05$ ). Subjects in group Xyls3 were older than those in the other groups (Table 2), while those in group Xyl-s5 were older than those of most other groups. The number of sound surfaces at risk among subjects in group $\mathrm{Xyl-s} 3$ was higher than that of other groups, except group Xyl-s5. The mean number of sound surfaces at risk in the "No gum" group and in group $X y 1-s 5$ was higher than that in groups Sugar-s5, 1:3XS-p5, and $X y 1-p 3$. The baseline DMFS score in group $X y 1-p 3$ was significantly lower than that for groups $\mathrm{Xyl}-\mathrm{s} 3$ and $\mathrm{Xyl}-\mathrm{s} 5$. There was significant heterogeneity among the groups with regard to gender distribution (chi-square with 8 degrees of freedom; $p=0.042$ ). Males comprised $54 \%$ of all subjects examined at baseline. Table 2 also gives the percentage of subjects in each group with evidence that they had been treated by a dentist (based on the number of fillings and extractions observed at baseline). The groups were divided into two categories: The "No-gum" group and groups Xyl-p3 and 1:3 XS-p5 had the lowest number of subjects who had seen a dentist, while all other groups were relatively similar in this regard.

\section{Dropouts}

Of the 1277 subjects examined at baseline, 416 were not seen at the last visit. The characteristics at baseline of those subjects who did not complete the 40-month study when compared with those who did were as follows: higher DMFS score (6.3 vs. 4.9 ), older (10.5 vs. 10.1$)$, and more sound surfaces ( 84.5 vs. 77.0 ). The dropout rate was related to the treatment groups (chi-square; $p<0.05$ ). For the sucrose and the sorbitol groups, it was lower than the mean dropout rate (11.8\% and $20.5 \%$, respectively, os. the average rate of $32.6 \%)$, and the dropout rate in group $\mathrm{Xyl}-\mathrm{s} 3$ was higher $(49.0 \%, \mathrm{p}<0.05)$ than the overall mean rate. There was no significant heterogeneity in dropout rate among the remaining six groups (chi-square; $\mathrm{p}=0.46$ ). 
Table 5. p-values associated with between-group comparisons ( 0 to 40 months), adjusted for gender, age, DMFS, and the number of sound surfaces at baseline

\begin{tabular}{|c|c|c|c|c|c|c|c|c|c|c|}
\hline Group & Abbreviation $^{a}$ & & & & & & & & & \\
\hline Number & Used in Text & 1 & 2 & 3 & 4 & 5 & 6 & 7 & 8 & 9 \\
\hline 1 & No gum & - & 0.1128 & 0.0074 & 0.0000 & 0.0000 & 0.0000 & 0.0000 & 0.0000 & 0.0000 \\
\hline 2 & Sugar-s5 & 0.1128 & - & 0.0000 & 0.0000 & 0.0000 & 0.0000 & 0.0000 & 0.0000 & 0.0000 \\
\hline 3 & Sorb-p5 & 0.0074 & 0.0000 & - & 0.0136 & 0.0013 & 0.0003 & 0.0000 & 0.0000 & 0.0000 \\
\hline 4 & $3: 2$ XS-p5 & 0.0000 & 0.0000 & 0.0136 & - & 0.3490 & 0.2223 & 0.0657 & 0.0346 & 0.0000 \\
\hline 5 & 1:3 XS-p5 & 0.0000 & 0.0000 & 0.0013 & 0.3490 & - & 0.8304 & 0.4331 & 0.2607 & 0.0003 \\
\hline 6 & Xyl-s3 & 0.0000 & 0.0000 & 0.0003 & 0.2223 & 0.8304 & - & 0.5435 & 0.3414 & 0.0004 \\
\hline 7 & Xyl-s5 & 0.0000 & 0.0000 & 0.0000 & 0.0657 & 0.4331 & 0.5435 & - & 0.6902 & 0.0027 \\
\hline 8 & $x y l-p 3^{b}$ & 0.0000 & 0.0000 & 0.0000 & 0.0346 & 0.2607 & 0.3414 & 0.6902 & - & 0.0130 \\
\hline 9 & Xyl-p5 $5^{b}$ & 0.0000 & 0.0000 & 0.0000 & 0.0000 & 0.0003 & 0.0004 & 0.0027 & 0.0130 & - \\
\hline
\end{tabular}

a Physical form of gum, i.e., stick (s) or pellet (p), and number of chewing episodes per day are indicated after hyphen; thus, -s5 means five episodes of chewing a stick gum.

b Marketed by Leaf Group B.V. as XyliFresh 100 and XyliFresh Professional. $\mathrm{n}=1135$.

\section{Examiner error analyses}

The inter-examiner reliability kappa statistics (Landis and Koch, 1977) of the four examiners for diagnosing a structurally intact surface $v s$. a cavitated surface were as follows: 0.96 at baseline, 0.88 at second visit, 0.86 at third visit, and 0.84 at fourth visit. The kappa statistic for all four visits combined was 0.91 . The intra-examiner reproducibility analyses (Shaw and Murray, 1975) for the above diagnoses gave figures that ranged-at the four examinations and for the four examiners-from 0.26 to 0.31 for $r$, and from 75 to 81 for $P$.

\section{Effects of gum use on caries increments}

A total of 1135 subjects was evaluated on at least two dates (Table 3). Among those subjects, a total of 3483 caries lesion onsets was observed on surfaces which were diagnosed as non-cavitated at baseline, or which erupted during the study. On average, there were 3.10 caries onsets/subject. The average number of onsets/subject ranged among the groups from a mean of 1.4 in group Xyl-p5 to a mean of 6.2 in group Sugar-s5. The average number of surface years of observation per subject was 301.3 during the entire study and ranged from a mean of 283.1 for group Xyl-p3 to a mean of 325.5 for group Sorb-p5 (Table 3). The structurally sound surfaces on which caries lesion onsets were diagnosed had the following overall maximum disease severity diagnosed during the 40 -month trial: $60.8 \% \mathrm{D}_{\mathrm{s}^{\prime}} 19.8 \% \mathrm{D}_{\mathrm{m}^{\prime}}$ and $11.0 \% \mathrm{D}_{\mathrm{x}}$.

During the 40-month study, the "No gum" control group had 15.9 lesion onsets per 1000 surfaces per year (Table 4). The Sugar-s5 group had a marginally significant increase in caries onset risk when compared with the control group

Table 6. Unadjusted mean estimates of DMFS scores at baseline and at 40 months for those 861 subjects seen at baseline and the last examination, and the matrix of pairwise comparison probabilities among all groups associated with adjusted $\triangle \mathrm{DMFS}$ scores

\begin{tabular}{|c|c|c|c|c|c|c|c|c|c|c|c|}
\hline $\begin{array}{l}\text { Group } \\
\text { Number }\end{array}$ & $\begin{array}{l}\text { Abbreviation }{ }^{a} \\
\text { Used in Text }\end{array}$ & $\mathrm{n}$ & $\begin{array}{l}\text { Score at } \\
\text { Baseline }\end{array}$ & $\begin{array}{l}\text { Score at. } \\
40 \text { Mos. }\end{array}$ & $\triangle \mathrm{DMFS}$ & $\triangle \mathrm{DMFS}^{\mathrm{b}}$ & $\begin{array}{l}\operatorname{Pr}>[\mathrm{T}]^{\mathrm{c}} \\
\text { Mean } \\
\triangle \mathrm{DMFS}=0\end{array}$ & 1 & 2 & 3 & $\mathrm{p}$-Values \\
\hline 1 & No gum & 89 & $4.3(0.9)$ & $9.3(0.9)$ & $5.0(0.5)$ & $4.9(0.5)$ & 0.0001 & - & 0.0202 & 0.0903 & 0.0001 \\
\hline 2 & Sugar-s5 & 109 & $4.7(1.0)$ & $11.2(1.0)$ & $6.5(0.5)$ & $6.6(0.5)$ & 0.0001 & 0.0202 & - & 0.0001 & 0.0001 \\
\hline 3 & Sorb-p5 & 120 & $5.4(0.7)$ & $9.1(0.7)$ & $3.7(0.4)$ & $3.8(0.4)$ & 0.0001 & 0.0903 & 0.0001 & - & 0.0155 \\
\hline 4 & $3: 2$ XS-p5 & 92 & $4.9(0.7)$ & $7.1(0.7)$ & $2.2(0.5)$ & $2.2(0.5)$ & 0.0001 & 0.0001 & 0.0001 & 0.0155 & - \\
\hline 5 & $1: 3$ XS-p5 & 83 & $3.8(0.6)$ & $4.5(0.6)$ & $0.6(0.5)$ & $0.6(0.5)$ & 0.4140 & 0.0001 & 0.0001 & 0.0001 & 0.0287 \\
\hline 6 & Xyl-s3 & 80 & $6.6(0.7)$ & $6.6(0.7)$ & $-0.05(0.5)$ & $0.1(0.5)$ & 0.9135 & 0.0001 & 0.0001 & 0.0001 & 0.0043 \\
\hline 7 & Xyl-s5 & 96 & $6.1(0.6)$ & $6.6(0.6)$ & $0.5(0.5)$ & $0.6(0.5)$ & 0.7015 & 0.0001 & 0.0001 & 0.0001 & 0.0204 \\
\hline 8 & Xyl-p3d & 97 & $3.4(0.4)$ & $4.3(0.5)$ & $0.8(0.5)$ & $0.9(0.5)$ & 0.1143 & 0.0001 & 0.0001 & 0.0001 & 0.0644 \\
\hline 9 & Xyl-p5 $5^{d}$ & 95 & $5.1(0.5)$ & $4.3(0.5)$ & $-0.7(0.5)$ & $-0.8(0.5)$ & 0.0228 & 0.0001 & 0.0001 & 0.0001 & 0.0001 \\
\hline
\end{tabular}

Physical form of gum, i.e., stick (s) or pellet (p), and number of chewing episodes per day are indicated after hyphen; thus, $-\mathrm{s} 5$ means five episodes of chewing a stick gum. Values are expressed as means, and standard errors are indicated in parentheses.

Adjusted for gender, age, and number of sound surfaces at baseline.

$\operatorname{Pr}>[T]$ Ho: $\operatorname{mean}(\mathrm{i})=$ mean $(\mathrm{j})$.

Marketed by Leaf Group B.V. as XyliFresh 100 and XyliFresh Professional. 
[20.0 lesion onsets per 1000 surfaces per year $(p=0.1128)$; relative risk, $1.20 ; 95 \%$ confidence interval, 0.96 to 1.49$]$. The caries onset risk for a surface in the Sorb-p5 group was $74 \%$ of that for a surface in the "No gum" group $(p=0.0074)$ (Tables 4 and 5). The caries onset risks in the 3:2 XS-p5 and $1: 3$ XS-p5 groups were only $56 \%$ and $49 \%$ of that in the "No gum" group (both $\mathrm{p}=0.0001$ ). There was no statistically significant difference between these two polyol mixture groups. The largest reductions in caries risk were observed in the groups receiving xylitol gums. The caries onset risks in the xylitol stick gum used either 3 or 5 times daily were only $48 \%$ and $44 \%$ of that for a surface of the "No gum" group (both $\mathrm{p}=0.0001$ ), while the caries onset risks for a surface in the xylitol pellet gum used either 3 or 5 times daily were further reduced to $41 \%$ and $27 \%$ of that for a surface in the "No gum" group (both $p=0.0001$ ).

Use of the xylitol-containing gums was associated with a significant reduction of caries onset rates compared with sucrose gum ( $p<0.0001)$ and sorbitol gum $(p<0.05)$. The Sorb-p 5 group had significantly reduced caries onset rates compared with Sugar-s5 ( $p<0.0001$ ) and "No gum" ( $p=$ $0.0074)$. Xyl-s 3 and Xyl-s5 use was associated with significantly fewer lesion onsets than Sorb-p5 ( $p=0.0003$ and $p<0.0001$, respectively). The Xyl-p5 group had significantly fewer lesion onsets than any non-xylitol group and also all other groups $(p=0.013$ ). The adjusted relative risk estimates were only moderately different from any of the less adjusted or unadjusted relative risk estimates (Table 4).

DMFS analyses were consistent with the conclusions regarding the relative superiority of the gums (Table 6). Accordingly, sorbitol gum use was associated with reduction of caries experience as compared with sugar gum $(p=0.0001)$. Gums containing mixtures of sorbitol and xylitol were associated with fewer lesion onsets than sorbitol or sugar gums, or "No gum". The gum group having the lowest caries onset rate was the xylitol pellet gum used five times daily.

\begin{tabular}{ccccc}
5 & 6 & 7 & 8 & 9 \\
\hline 0.0001 & 0.0001 & 0.0001 & 0.0001 & 0.0001 \\
0.0001 & 0.0001 & 0.0001 & 0.0001 & 0.0001 \\
0.0001 & 0.0001 & 0.0001 & 0.0001 & 0.0001 \\
0.0287 & 0.0043 & 0.0204 & 0.0644 & 0.0001 \\
& 0.5005 & 0.9684 & 0.6728 & 0.0476 \\
0.5005 & - & 0.4986 & 0.2671 & 0.2207 \\
0.9684 & 0.4986 & - & 0.6348 & 0.0453 \\
0.6728 & 0.2671 & 0.6348 & - & 0.0125 \\
0.0476 & 0.2207 & 0.0453 & 0.0125 & - \\
\hline
\end{tabular}

\section{Discussion}

The above data indicate that chewing gum usage can substantially affect caries risk. These results also suggest that a relationship between xylitol concentrations and caries rate existed: Usage of sorbitol-containing but xylitol-free gum was associated with some reduction of caries experience; use of the two xylitol/sorbitol mixture gums was associated with a greater reduction, and use of the xylitol-containing but sorbitol-free gums was associated with the greatest caries reduction. Possible biochemical explanations of these effects have been discussed (Mäkinen, 1989) and will be further elucidated in ancillary plaque and saliva studies of the present subjects (to be published). Variation of caries rates over time (period effects) will also be reported separately.

The goal of the analyses was to provide the best defense of the hypothesis of no association between the chewing gum regimens and caries lesion onset (Maclure, 1990). First, crude or unadjusted differences were presented, these not taking into account baseline differences which existed between groups. As a result, it could be that the observed differences were not a result of treatment assignments, but that they were a consequence of baseline inequalities. Subsequently, adjusted analyses were presented which sought to remove any distortion introduced by inequalities in baseline covariates between treatment groups (Anderson et al., 1980). Adjustment of the analyses for gender, patient's age, initial caries experience, or the number of sound surfaces at risk at baseline had only a minimal effect on the reported estimates.

The human subjects review panel of the University of Michigan and the Ministry of Health of Belize considered the scientific benefits to be gained by the inclusion of the sugar gum in the protocol to outweigh any risks from the inclusion of a sugar gum group in the study design in a society with a very high sugar intake. The present results showed that the caries rates differed marginally between the sugar group and the "No gum" group, probably because the overall sugar exposure and frequency of use were comparable for both groups. Of course, it was impossible to know to what extent the "No gum" group consumed sugars (including sugar-containing gum) which can be purchased readily in Belize.

Confounding variables which were not measured at baseline cannot be adjusted for, and as a result, cohort studies are less reliable for the detection of treatment effects than are randomized controlled trials. When the results of clinical studies are evaluated, it is important to consider the size of the observed treatment effect and to evaluate whether the observed treatment effect could be the result of biases introduced by unknown confounding variables. Some of the treatment effects observed in this study are of such magnitude (e.g., a relative risk of 0.27 ) that it may be difficult to assume that such an effect was the result of bias introduced by unknown confounding variables present at baseline. The observed dose-response effects, the biological plausibility, and the fact that this study confirmed previous study findings should increase confidence. Of course, it is possible that some unknown confounding variable is responsible for the study findings. The schools constitute one such variable. During the conduct of the study, it became apparent that socio-economic differences may have 
existed between schools. Consequently, it is also possible that differences in oral hygiene practices existed between pupils who went to different schools. Such differences may have been responsible for baseline inequalities in DMFS scores and also in inequalities in the number of subjects with evidence of dental care at baseline. Even though adjustment for DMFS analyses did not affect the study findings, it is possible that some other unknown confounding variables were associated with both the treatments and the risk for caries onset. It is also possible that the unbalanced losses of subjects in follow-ups introduced a potential bias, necessitating a cautious interpretation of the results and validation of the results in other populations.

The a priori decision to perform this study for 40 months with initially 10-year-old children resulted from the known caries risk during this age, the impending eruption of second molars and premolars during the period of the study, the obligation of such children to attend the compulsory public elementary schools of Belize City, and the recognition that following elementary school, only about $50 \%$ of pupils continue their education at high schools. The five-minute supervised chewing practice was chosen to clarify the impact of the sweeteners on caries, as distinct from the effect of chewing alone (surveys suggested, however, that the chewing time on non-school days was from 10 to $30 \mathrm{~min}$ ). Data from the authors' laboratory indicate that most of the carbohydrates found in gum leach into saliva within $5 \mathrm{~min}$, and the literature indicates that the maximal plaque-pH-lowering effect of fermentable carbohydrate consumption in the form of gum is reached between 3 and $7 \mathrm{~min}$ (Stephan, 1944; Imfeld, 1977). The reason for the superiority of the pellet-shaped gums is not known. However, pellet and stick gums have a different gum base, and the pellets are additionally coated with the corresponding pure carbohydrate sweetener, factors which may contribute to the gums' relative effectiveness. Further studies are needed to elucidate this question.

The dropout rate of $32.6 \%$ in this study was anticipated. Dropout rates in school-based chewing gum studies are high in general, three other large-scale chewing gum studies with follow-up periods significantly shorter than that of the present study having had dropout rates of $53 \%$ (Kandelman and Gagnon, 1990), 37.3\% (Kandelman et al., 1988), and 30.4\% (Scheinin et al., 1985a,b). At the 28-month examination period in the present study, the dropout rate was $25.7 \%$. No adverse sideeffects were reported, and the reasons given by teachers for dropout were congruent with the expected demographic behavior of the study population. Dropout rates even in supervised classroom studies are typically substantial, e.g., during a five-year study of American children treated with fluoride rinses, fluoride tablets, or a combination, dropout rates were about $52 \%$ (Driscoll et al., 1990).

Although the DMFS analyses were consistent with the caries rate data, the caries risk values (Table 4) seem to describe the overall caries onset rate more accurately, the tooth surfaces being at risk in all groups. The DMFS scores reflect the difficulties of integrating the caries experience.

There is wide agreement that frequent consumption of sucrose and other easily fermentable carbohydrates is the major dietary factor in the development of dental caries. The specific relationship between dietary sugars (certain mono- and disaccharides) and dental caries has been recognized for decades (Mandel, 1970; Sreebny, 1982; Rugg-Gunn, 1983; Ismail et al., 1984; Burt, 1986; Loesche, 1986; Tanzer, 1993, 1995), and the present study supports this relationship. The present data also suggest, consistent with earlier findings (Scheinin et al., 1975), that habitual, long-term use of sugar gum may not prevent caries, despite the possible benefit of salivary stimulation by gum chewing; further studies with longer chewing times might show some increase in salivary benefits. The results indicate that decreases in caries rates that are induced by including a polyol- and preferably a xylitol-based chewing gum program in basic caries prevention can be substantial and clinically meaningful, even when the consumption of sugary items is high and subjects have limited access to other preventive or restorative dental treatment.

\section{Acknowledgments}

We thank Dr. Theodore Aranda and The Hon. Reuben Campos, previous and succeeding Ministers of Health of Belize, respectively; the Permanent Secretaries of Health and Directors of Health Services of Belize; and all participating school principals and teachers. Special thanks to the children of Belize City and their families. The generous financial support of Leaf Group (Amsterdam, The Netherlands; Lake Forest, IL; and Turku, Finland), a division of Huhtamäki Oy (Helsinki, Finland), is acknowledged. The Finnish Cultural Fund, The Finnish Independence Jubilee Fund, the University of Michigan, University of Turku, and the Ylivieska Public Health Centre, Ylivieska, Finland, also provided assistance. Mr. Sheldon Siegel, Vice-President for Research and Development, Leaf Group, is offered sincere thanks for long-term collaboration, patience, and trust. We are also indebted to Drs. Richard Brogle, Chin-Yu Chen, Irwin Mandel, Eva Söderling, and Frans Toors for their criticism of study design, execution, and evaluation. The technical assistance of Irma Bennet, Aino Isokangas, Riitta Isotupa, Kitty Kahn, Inez Klein, and Diana Pape is gratefully acknowledged, as is the assistance of Robert Huzinec, Virva Mäkelä, Greg Motto, and Sakari Taskinen of the gum manufacturing plants.

\section{References}

American Dental Association (1992). The 1991 survey of dental practice. General characteristics of dentists. Chicago, IL.

Anderson S, Auquier A, Hauck WW, Oakes D, Vandaele W, Weisberg HI (1980). In: Statistical methods for comparative studies: techniques for bias reduction. New York: Wiley.

Belize Ministry of Finance-Central Statistical Office (1991). 1991 Population census. Central Statistical Office. Government Printery, Belmopan, Belize.

Bennett CA (1989). Clinical national oral health survey (Knowledge, attitude and practice survey; KAP survey), Ministry of Health, Belize, Belmopan.

Birkhed D (1994). Cariologic aspects of xylitol and its use in chewing gum: A review. Acta Odontol Scand 52:116-127.

Breslow NE, Day NE (1980). Fundamental measures of disease occurrence and association. In: Statistical methods in cancer research. Vol. 1. The analysis of case-control studies. Lyon, France: International Agency for Research on Cancer.

Brunelle JA (1989). Dental caries of United States children. The national survey of caries in U.S. schoolchildren: 1986-1987. 
National survey and regional findings. Washington, DC US Dept. of Health and Human Services. NIH Pub No 89 2247, pp. 1-337.

Burner ST, Waldo DR, McKusick DR (1992). National health expenditures projections through 2030. Health Care Finan Rev 14:1-29.

Burt BA (1986). Community-based methods for preventing dental caries and periodontal disease. In: Systematized prevention of oral disease: theory and practice. Granath L, McHugh WD, editors. Boca Raton, FL: CRC Press, pp. 145-160.

Charbeneau GT (1988). In: Principles and practice of operative dentistry. 3rd ed. Philadelphia: Lea and Febiger, pp. 26, 27.

Driscoll WS, Nowjack-Raymer R, Heifetz SB, Li S-H, Selwitz RH (1990). Evaluation of the comparative effectiveness of fluoride mouthrinsing, fluoride tablets, and both procedures in combination. Interim findings after five years. I Publ Health Dent 50:13-17.

Glass RL (1981). Effects on dental caries incidence of frequent ingestion of small amounts of sugars and stannous EDTA in chewing gum. Caries Res 15:256-262.

Glinsmann WH, Irausquin H, Park YK (1986). Evaluation of health aspects of sugars contained in carbohydrate sweeteners. / Nutr 116(Suppl 11):S1-S216.

Hujoel PP, Isokangas PJ, Tiekso J, Davis S, Lamont RJ, DeRouen TA, et al. (1994). A re-analysis of caries onset rates in a preventive trial using Poisson regression models. J Dent Res 73:573-579.

Imfeld T (1977). Evaluation of the cariogenicity of confectionery by intra-oral wire-telemetry. Helv Odont Acta 21:1-28.

Ismail AI, Burt BA, Eklund SA (1984). The cariogenicity of soft drinks in the United States. I Am Dent Assoc 109:241-245.

Isokangas P, Alanen P, Tiekso J, Mäkinen KK (1988). Xylitol chewing gum in caries prevention: a field study in children. J Am Dent Assoc 117:315-320.

Kandelman D, Gagnon G (1990). A 24-month clinical study of the incidence and progression of dental caries in relation to consumption of chewing gum containing xylitol in school preventive programs. J Dent Res 69:1771-1775.

Kandelman D, Bär A, Hefti A (1988). Collaborative WHO xylitol field study in French Polynesia. I. Baseline prevalence and 32month caries increment. Caries Res 22:55-62.

Karim MR, Zeger SL (1988). GEE: A SAS macro for longitudinal data analysis. Technical report \#674. Department of Biostatistics. Baltimore, MD: The Johns Hopkins University.

Landis JR, Koch GG (1977). The measurement of observer agreement for categorical data. Biometrics 33:159-174.

Liang KY, Zeger SL (1986). Longitudinal data analysis using generalized linear models. Biometrika 73:13-22.

Licht FO (1993). Sugar and sweetener yearbook. World sugar statistics. London: International Sugar Organization; Rome: Food and Agriculture Organization.

Lilienfeld DE, Stolley PD (1994). Foundations of epidemiology. 3rd ed. New York: Oxford University Press.

Loesche WJ (1986). Role of Streptococcus mutans in human dental decay. Microbiol Rev 50:353-380.

Louis TA (1988). General methods for analyzing repeated measures. Statistics Med 7:29-45.

Maclure M (1990). Multivariate refutation of aetiological hypotheses in non-experimental epidemiology. Int J Epidemiol 19:782-787.

Mäkinen KK (1989). Latest dental studies on xylitol and mechanism of action of xylitol in caries limitation. In: Progress in sweeteners. Grenby $\mathrm{TH}$, editor. London:
Elsevier, pp. 331-362.

Mandel ID (1970). Effects of dietary modifications on caries in humans. J Dent Res 49(Spec Iss):1201-1211.

McCullagh P, Nelder JA (1989). Generalized linear models. 2nd ed. London and New York: Chapman and Hall.

Miller AJ, Brunelle JA, Carlos JP, Brown LJ, Löe H (1987). Oral health of United States adults. The national survey of oral health in U.S. employed adults and seniors: 1985-1986 national findings. Washington, DC: US Dept. of Health and Human Services, NIH Pub No 87-2868, pp. 1-168.

Möller II, Poulsen S (1973). The effect of sorbitol-containing chewing gum on the incidence of dental caries, plaque and gingivitis, in Danish school children. Community Dent Oral Epidemiol 1:58-67.

Newbrun E (1989). Cariology. 3rd ed. Chicago: Quintessence Publishing Company, pp. 248-252.

Pape HR Jr, Mäkinen KK (1994). Verification and maintenance of dental explorer sharpness. Oper Dent 19:221-223.

Radike AW (1972). Criteria for diagnosis of dental caries. In: Proceedings of the conference on the clinical testing of cariostatic agents. Chicago, IL: American Dental Association, p. 87.

Rugg-Gunn AJ (1983). Diet and dental caries. In: Prevention of dental disease. Murray JJ, editor. Oxford: Oxford University Press, pp. 3-82.

Scheinin A, Mäkinen KK, Tammisalo E, Rekola M (1975). Incidence of dental caries in relation to 1-year consumption of xylitol chewing gum. Acta Odontol Scand 33(Suppl 70):307-316.

Scheinin A, Bánóczy J, Szöke J, Esztári I, Pienihäkkinen K, Scheinin U, et al. (1985a). Collaborative WHO xylitol field studies in Hungary. I. Three-year caries activity in institutionalized children. Acta Odontol Scand 43:327-347.

Scheinin A, Pienihäkkinen K, Tiekso J, Bánóczy J, Szöke J, Esztári I, et al. (1985b). Collaborative WHO xylitol field studies in Hungary. VII. Two-year caries incidence in 976 institutionalized children. Acta Odontol Scand 43:381-387.

Shaw L, Murray JJ (1975). Inter-examiner and intra-examiner reproducibility in clinical and radiographic diagnosis. Int Dent J 25:280-288.

Sreebny LM (1982). Sugar and human dental caries. World Rev Nutr Diet 40:19-65.

Stephan RM (1944). Intra-oral hydrogen-ion concentrations associated with dental caries activity. J Dent Res 23:257-266.

Stephen KW (1993). Caries in young populations-worldwide. In: Cariology for the nineties. Bowen WH, Tabak LA, editors. Rochester: University of Rochester Press, pp. 37-50.

Sturdevant CM (1995). In: The art and science of operative dentistry. 3rd ed. St. Louis: Mosby, pp. 89-97, 203, 295.

Tanzer JM (1993). Sweeteners and dental caries. Some emerging issues. In: Cariology in the nineties. Bowen W', Tabak LA, editors. Rochester: University of Rochester Press, pp. 383-396.

Tanzer JM (1995). Xylitol chewing gum and dental caries. Int Dent J 45(Suppl 1):65-76.

Woodward M, Walker ARP (1994). Sugar consumption and dental caries, evidence from 90 countries. Br Dent J 176:297-302.

World Health Organization (1979). A guide to oral health epidemiological investigations. Geneva: WHO.

World Health Organization (1984). Technical report series 713: Prevention methods and programmes for oral diseases. Geneva: WHO.

World Health Organization (1989). Dental caries levels at 12 years (mimeograph of data from global data bank). Geneva: WHO. 